\title{
Editorial
}

\section{Deployment Challenges for Large-Scale MIMO Communications}

\author{
Ahmed Iyanda Sulyman, ${ }^{1}$ Joonhyuk Kang, ${ }^{2}$ and Shahram Yousefi ${ }^{3}$ \\ ${ }^{1}$ Department of Electrical Engineering, King Saud University, Riyadh 11421, Saudi Arabia \\ ${ }^{2}$ Department of Electrical Engineering, KAIST, Daejeon 305-702, Republic of Korea \\ ${ }^{3}$ Department of Electrical and Computer Engineering, Queen's University, Kingston, ON, Canada K7L 3N6
}

Correspondence should be addressed to Ahmed Iyanda Sulyman; asulyman@ksu.edu.sa

Received 10 September 2014; Accepted 10 September 2014; Published 9 November 2014

Copyright (C) 2014 Ahmed Iyanda Sulyman et al. This is an open access article distributed under the Creative Commons Attribution License, which permits unrestricted use, distribution, and reproduction in any medium, provided the original work is properly cited.

Large-scale (or massive) MIMO systems have been shown to hold great potential for realizing Gigabit data rates in the next generation wireless networks. When networks deploy massive MIMO-enabled base stations (BS) or access points, their capacity can be enhanced by as much as tenfold (or more), compared to the existing systems. This extra degree of freedom, offered by massive MIMO systems, could be used to expand current system capacity by bringing in more users in the system or to increase the individual user's data rate/throughput.

Capacity gains for single-user MIMO system can be scaled up roughly from 6 bits per seconds $(\mathrm{bps}) / \mathrm{Hz}$ to $16 \mathrm{bps} / \mathrm{Hz}$ using large-scale single-user MIMO system with 100 antenna elements, at a relative energy efficiency of $10^{-1}[1]$. If massive multiuser MIMO systems with same 100 antenna elements are used, however, the capacity can be scaled up by as much as $60 \mathrm{bps} / \mathrm{Hz}$ [1]. This is a significant capacity boost that has attracted many in the wireless industry. Figure 1 illustrates the capacity curves for massive multiuser MIMO systems in comparison with the single-user MIMO case, where it is easy to observe the increased capacity gain achieved in massive multiuser MIMO case when serving large number of users simultaneously. This is the key benefit of massive MIMO systems over the traditional ones. Unlike the previous cases where significant capacity boosts were achieved via the deployments of new access or modulation schemes, the capacity boost achievable using massive MIMO systems can be realized with existing technologies by simply scaling up the numbers of antenna elements at the BS and developing the associated software updates. For this reason, researchers and system developers have devoted significant attentions to the development of massive multiuser MIMO techniques lately.

Recently also, wireless system developers have been exploring possible upgrade of the existing wireless systems to higher frequency bands in the Millimeter Wave (mmWave) spectrum $(30-300 \mathrm{GHz})$ [2]. As shown in Figure 2, there is a massive amount of bandwidth currently available in this band with light use.

It is observed recently in [3] that despite the lossy nature of the mm-Wave channels compared to their microwave ( $\mu$ Wave) counterparts operating below $5 \mathrm{GHz}$, future wireless systems operating in the $\mathrm{mm}$-Wave bands can in fact be made more reliable than today's systems when highly directional steerable antennas and beam-forming techniques are deployed at the BS and mobiles. At the mm-Wave frequencies, the wavelength is so small that hundreds of antenna elements can be designed on the same physical spaces where only a few elements are currently possible. It is therefore been generally observed that $\mathrm{mm}$-Wave communications will play a key role in facilitating the deployments of massive MIMO systems in the upcoming wireless systems. The IEEE 802.11ad (WiGig) extension of the Wi-Fi standard has just been released. It operates in the unlicensed $60 \mathrm{GHz}$ bands of the mm-Wave spectrum, and it will allow the development of wireless local area networks (WLAN) devices capable of delivering $7 \mathrm{Gbps}$ data rate over the Wi-Fi network. The cellular networks will similarly embrace high frequency upgrade to the $\mathrm{mm}$ Wave bands in the upcoming fifth-generation (5G) systems. Cellular carriers world-wide are likely to utilize the $28 \mathrm{GHz}$, $38 \mathrm{GHz}$, and $73 \mathrm{GHz}$ bands of the mm-Wave spectrums in their 5G networks [3]. They will take advantage of the huge 


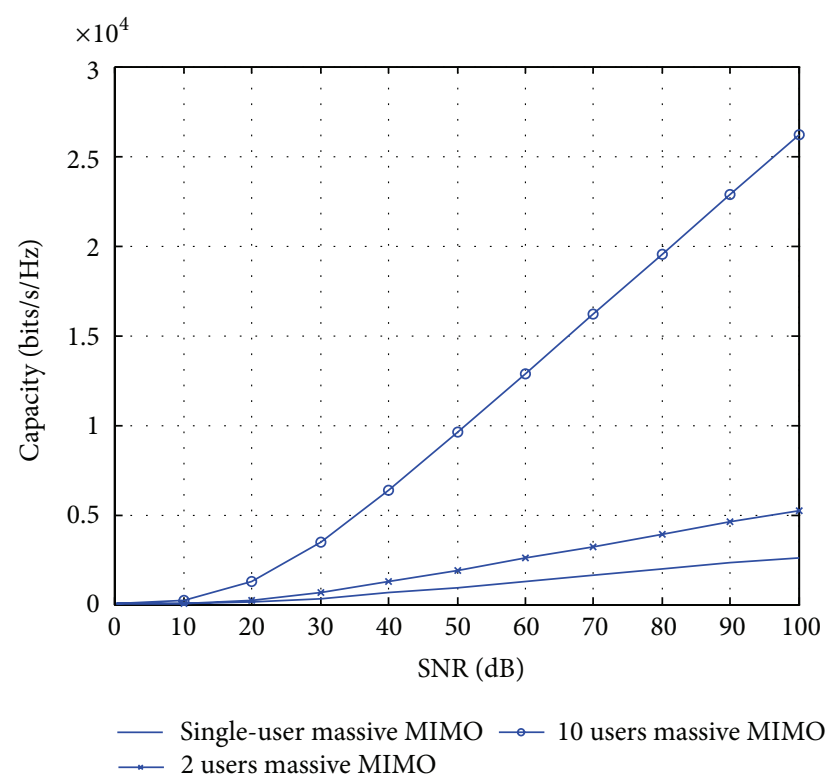

FIgURE 1: Capacity curve for large scale multiuser MIMO systems with 100 antenna elements at the BS.

bandwidth available in these bands to realize the $5 \mathrm{G}$ vision of delivering Gigabit data rates from BS on to mobile devices. Moving up to these high frequency bands will facilitate the deployments of aggressively large numbers of antennas (perhaps hundreds or thousands) on BS and possibly a few tens of antennas on mobile devices, all of which had hitherto been deemed impractical. Practical deployments of large-scale MIMO systems thus look increasingly more attractive in the light of the current shifts towards mm-Wave communications in the wireless industry.

Three manuscripts in this issue address different aspects of system designs for future mm-Wave communication systems employing large-scale antenna arrays. The paper by J. S. Kim et al. develops a central network architecture for $5 \mathrm{G}$ cellular systems employing massive antenna structures over mm-Wave bands. Their proposed network architecture utilizes $1 \mathrm{GHz}$ bandwidth in the $27 \mathrm{GHz}$ mm-Wave band to deliver ultrahigh system capacity on the order of hundred Gbps, to support various multimedia services and huge traffic expected to be generated in $5 \mathrm{G}$ networks. This is accompanied by two other manuscripts on the design of compact MIMO antennas operating in or near the mmWave spectrum. N. K. Kiem et al. present a compact $4 \times 4$ MIMO antenna operating in the frequency bands $2.73 \mathrm{GHz}$ to $10.68 \mathrm{GHz}$, which covers entirely the ultrawide band (UWB) frequency range of 3.1 to $10.6 \mathrm{GHz}$. The designed antenna also provides significant band rejection at the WLAN frequency of 5.36-6.04 GHz in order to help reduce interference placed on existing Wi-Fi systems operating in the $5 \mathrm{GHz}$ bands, when their antenna is deployed. T. Cella et al. present the design of a compact mm-Wave MIMO system with optimized capacity and phased arrays at the $70 \mathrm{GHz}$ mm-Wave bands. Their system is shown to achieve capacity up to $29 \mathrm{bps} / \mathrm{Hz}$ at a transmitter-receiver separation distance of $200 \mathrm{~m}$.
Massive multiuser MIMO systems typically employ precoding techniques to cancel the multiuser interference generated when a BS communicates simultaneously with multiple users on the same time-frequency resources. Maximumratio transmission (MRT) and zero-forcing (ZF) precoders have been widely explored for this application due to their simplicity of implementations and overall good performance. Spatial modulation is another emerging transmission technique that utilizes the antenna indexes, in a multiple antenna system, as additional means of data transmission besides the modulated symbols. The paper by K. M. Humadi et al. develops a receiver-side spatial modulation scheme that utilizes the user's subchannel index corresponding to the $\mathrm{ZF}$ precoding matrix employed at the BS, to convey extra useful information to the users in addition to the transmitted modulated symbol. Their results indicate that significant throughput enhancement is achievable using this approach.

Although massive multiuser MIMO system will provide significant capacity boosts, their deployments will also come with new challenges associated with the practical difficulties of deploying very large number of antenna elements on one single BS or mobile device and keeping all of them in working conditions all the time. When some of the antenna elements fail to work during operation, the data fed on to them is lost in the wireless transmission medium. This gives rise to the so-called wireless erasure channel or lossy links. The performance of massive MIMO systems with such lossy links will not be the same as the ones without losses. Special techniques to cope with these losses, whenever they arise, may therefore be needed to be incorporated in the design of massive MIMO systems. This is an important practical consideration that has not yet been raised in any previous article in the literature. A. H. Alqahtani et al. examine the performance of massive MIMO systems in the light of this problem. They show that the regular precoders used in massive MIMO systems are unable to completely remove the multiuser interference in a lossy wireless link, degrading the overall system performance. They then illustrate the use of rateless space-time codes (STC), to combat these losses. Their results indicate that up to $25 \%$ losses in the links can be tolerated when using rateless STC with large scale multiuser MIMO systems.

The performance of massive MIMO systems is heavily influenced by the underlying beamforming technique used for the multiuser data access. Several works have thus been devoted to the design of simple and effective beamforming algorithms for the multiuser access problem in massive multiuser MIMO system. Dr. M. Hefnawi develops here an adaptive beamforming algorithm for large-scale multiantenna systems using the concept of OFDM-SDMA. The proposed algorithm allows multiple users to be serviced on the same OFDM subchannel, in the context of cognitive radio system where the uplink MIMO channel capacity for a cognitive (secondary) user is maximized while keeping the interference levels placed on to the primary spectrum user below certain desired value.

To complete this issue, two articles are devoted to the subject of antenna subset selections. This problem arises whenever there is a large number of antenna elements 


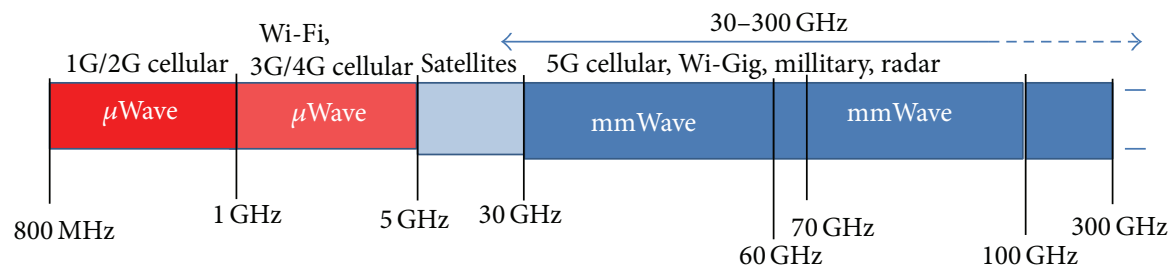

FIGURE 2: An overview of the mm-Wave Spectrum for future multiantenna wireless communications.

available at the RF stage, and limited hardware resources at base-band prevents the processing of all the signals received from the antennas. It is widely speculated that this problem may also be encountered in massive MIMO systems, since there are hundreds of antenna elements to be deployed and not all of them may need to be processed every time. A smart antenna selection strategy that captures most of the benefits of massive MIMO systems while processing only subsets of the available signals is, therefore, very essential in this situation. Q. Wang and H. Yang present a "group switch-andexamine" combining scheme for antenna selection problem in massive MIMO system. Their approach provides better effective capacity at lower operational complexity, compared to the existing antenna selection methods, when used in massive MIMO systems. This is followed by the paper by K. Qian et al., which presents low-complexity optimization method for the design of joint transmit antenna selection and beamforming techniques in large-scale MIMO systems.

By compiling these papers, we hope to enrich our readers and researchers with respect to the prospects and challenges of massive MIMO systems in the next generations of wireless systems.

\section{Ahmed Iyanda Sulyman Joonhyuk Kang Shahram Yousefi}

\section{References}

[1] E. G. Larsson, O. Edfors, F. Tufvesson, and T. L. Marzetta, "Massive MIMO for next generation wireless systems," IEEE Communications Magazine, vol. 52, no. 2, pp. 186-195, 2014.

[2] Z. Pi and F. Khan, "An introduction to millimeter-wave mobile broadband systems," IEEE Communications Magazine, vol. 49, no. 6, pp. 101-107, 2011.

[3] A. I. Sulyman, A. T. Nassar, M. K. Samimi, G. R. MacCartney Jr., T. S. Rappaport, and A. Alsanie, "Radio propagation path loss models for $5 \mathrm{G}$ cellular networks in the $28 \mathrm{GHZ}$ and $38 \mathrm{GHZ}$ millimeter-wave bands," IEEE Communications Magazine, vol. 52, no. 9, pp. 78-86, 2014. 

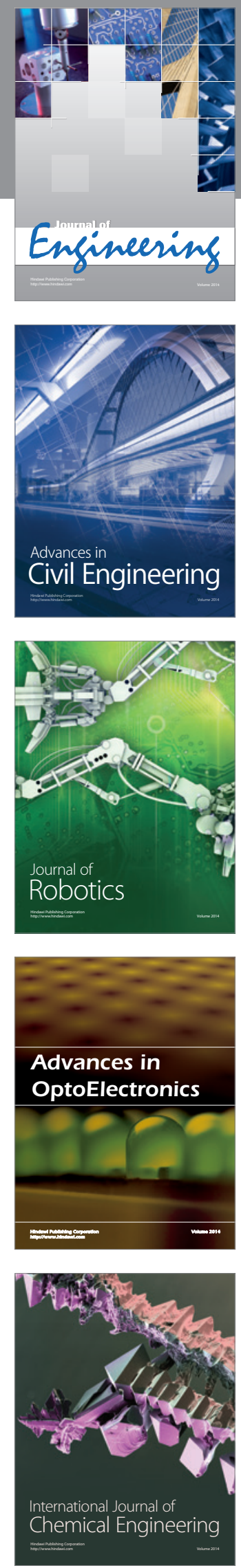

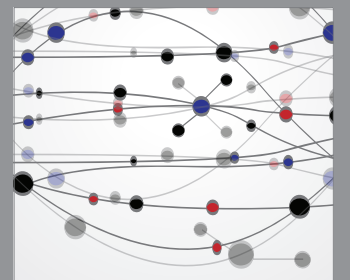

The Scientific World Journal
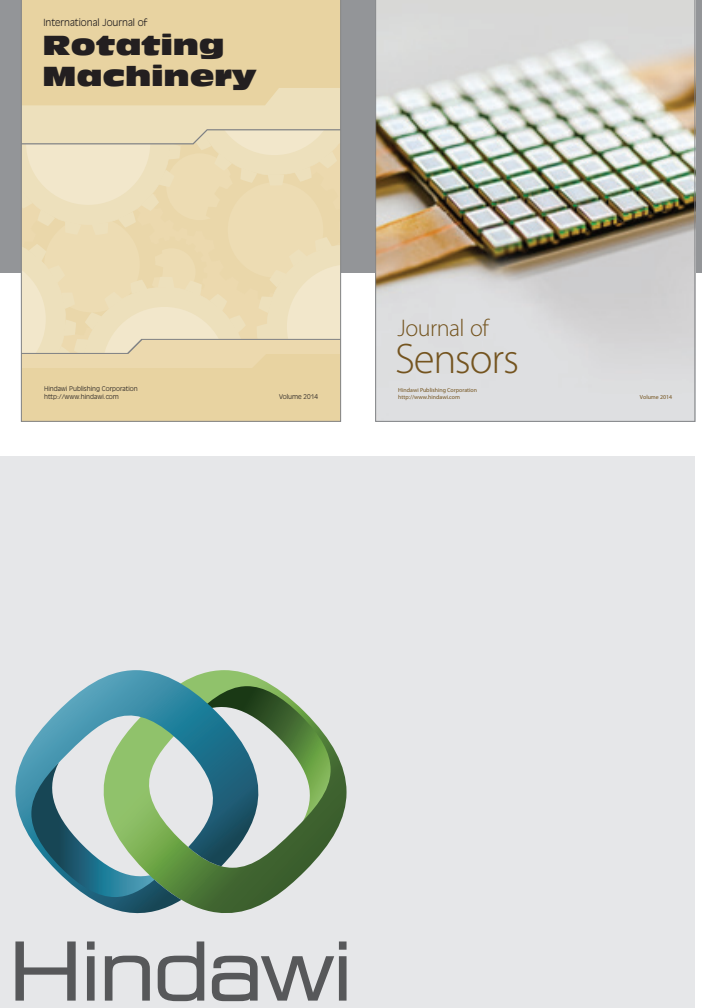

Submit your manuscripts at http://www.hindawi.com
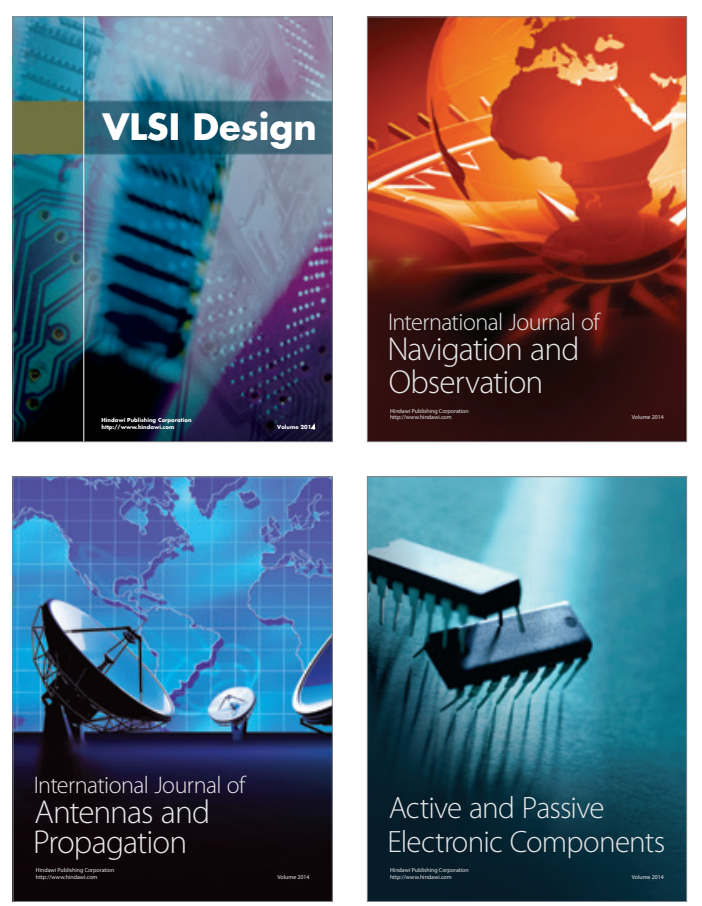
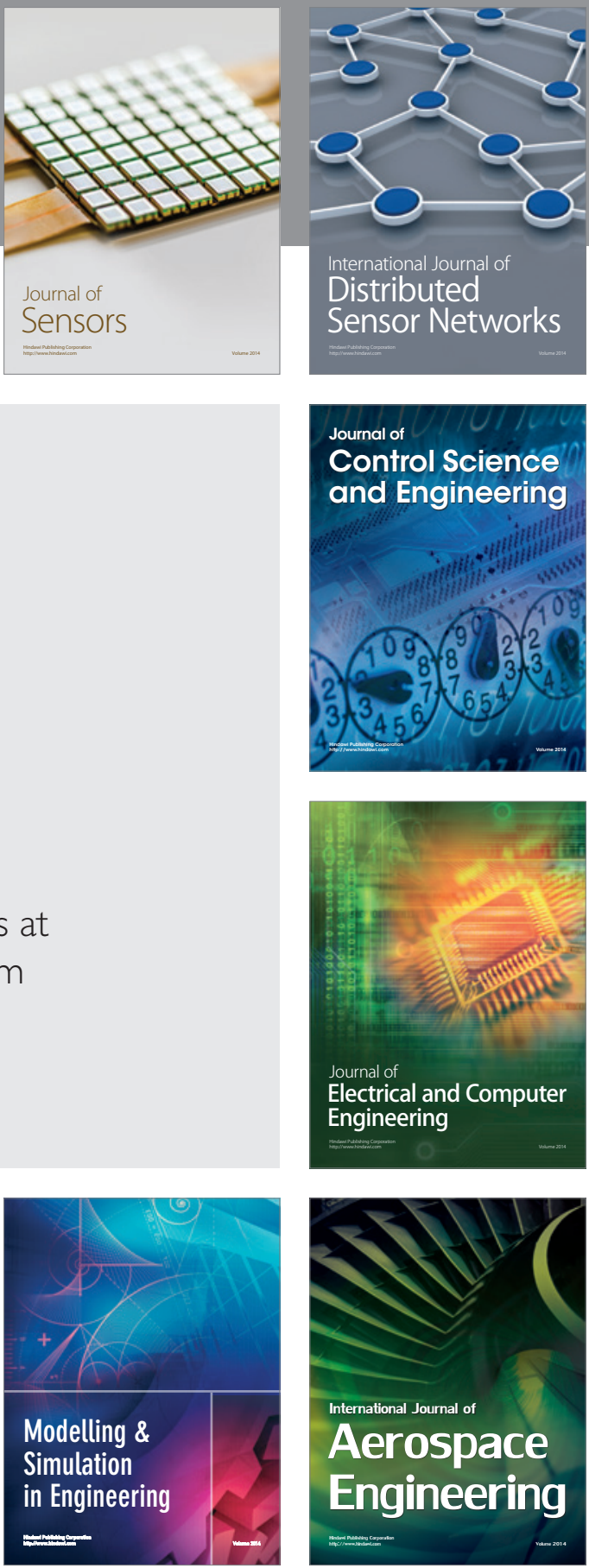

Journal of

Control Science

and Engineering
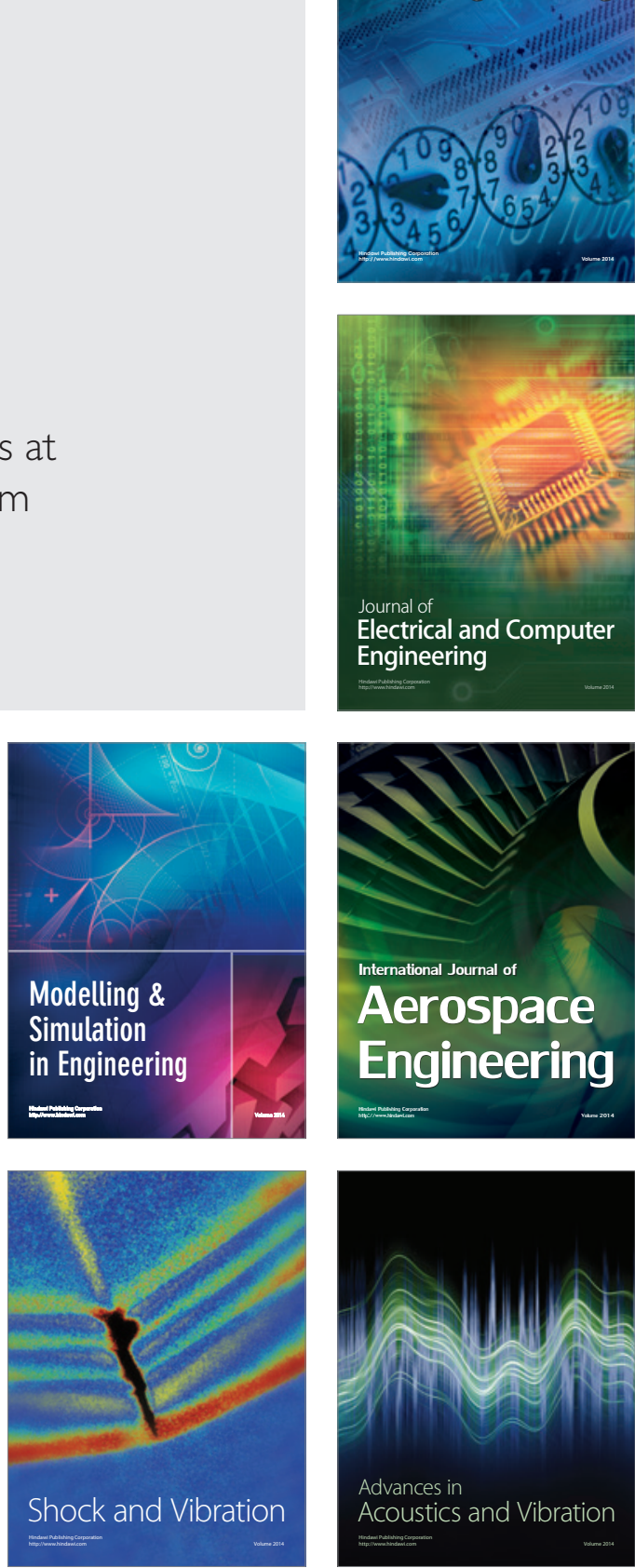